\title{
Bickerstaff Brainstem Encephalitis. A Rare Entity in Children
}

\author{
Muhammad Saeed*, Mian Tahir Ali Shah \\ Armed Forces Hospital, Khamis Mushayt, Saudi Arabia.
}

\begin{abstract}
Fisher and Bickerstaff, in the 1950s, reported some cases with distinctive features of ophthalmoplegia and ataxia. Bickerstaff's Brainstem Encephalitis (BBE) is an uncommon nervous system disorder characterized by clinical signs and symptoms including acute ophthalmoplegia, ataxia, and impaired consciousness. In Bickerstaff brainstem encephalitis, hyperreflexia was noted which support of a central pathology.
\end{abstract}

Keywords: Bickerstaff Brain Stem Encephalitis (BBE), Hyperreflexia, IVIG (Intravenous immunoglobulin), Magnetic Resonance Imaging (MRI), CSF PCR, Autoimmune, Anti-GQ1b antibody.

\section{INTRODUCTION}

Bickerstaff brainstem encephalitis (BBE), was reported first time in 1951, is an uncommon post-infectious central nervous system disorder diagnosed clinically by ophthalmoplegia, ataxia and altered conscious level, hyperreflexia, and up going plantar responses [1]. The cases reported with Bickerstaff brainstem encephalitis were having disturbed conscious level and in some cases, association of hyperreflexia was also reported, which support a central pathology. In 1957, Bickerstaff went on to expand his case series [2].

The exact incidence and prevalence of BBE in the world is not well known but in Japan it has been reported higher as compare to Western world. A recent report from Japan with $\mathrm{BBE}$ estimated that the annual incidence of $\mathrm{BBE}$ is approximately $0.078 / 100,000$ population [3].

$\mathrm{BBE}$ is supposed to be a post-infectious disorder in majority of the cases but can occur without any preceding infections or illness. The Anti-GQ1b, antibody may have a role in the pathogenesis of BBE, however the exact underlying cause has yet to be discovered. Disturbance of conscious level is a key feature which helps to distinguish BBE from MFS and GBS.

Different treatments have been reported for BBE, which include; 1) corticosteroids \& plasma exchange, 2) plasmapheresis without corticosteroids, 3) corticosteroids only, 4) both corticosteroids \& intravenous immunoglobulin (IVIG) \& plasma exchange, and 5) comprehensive immunotherapy [4]. Here we report a case with anti-GQ1b antibody- positive BBE who was successfully treated with IVIG.

\section{CASE REPORT}

A previously well 9-year-old boy presented to our emergency department with complaints of unsteady gait, followed by

*Address correspondence to this author at the Armed Forces Hospital, Khamis Mushayt, Saudi Arabia. E-mail: muhammad964@hotmail.com generalized weakness with slurred speech and difficulty in swallowing for 2 days and four hours history of altered sensorium. There was history of fever, cough and vomiting five days before presentation, for which he was treated in other hospital. There was no history of diplopia, or neck stiffness, convulsions, skin rash, no Bowel or bladder dysfunction, exposure to toxic materials, head injury, headache, or recent travelling.

On examination, his conscious level was altered but vital signs were stable. There were no signs of meningeal irritations. On cranial nerve examination, he had left sided upper motor neuron facial weakness and he was unable to close his left eye completely. His pupils were normal equal in size and symmetrically reactive to light. His motor system examination revealed, normal muscle bulk, tone but power was decreased both in upper and lower limbs $4 / 5$ in upper limbs and 3/5 in lower limbs respectively. He was able to walk with support but with unsteady wide based gait. His deep tendon reflexes in the lower limbs were exaggerated DTR +3 . Babinski sign was positive, planter response extensor. Rest of the systemic examinations were unremarkable.

His routine investigations $\mathrm{CBC}$ and serum chemistry results were unremarkable. Cerebrospinal fluid (CSF) examination revealed $2 \mathrm{WBC} / \mathrm{mm} 3$ (all lymphocytes), protein of 180 $\mathrm{mg} / \mathrm{dL}$ and glucose of $3.8 \mathrm{mmol} / \mathrm{L}$. CSF culture and PCR panel for virus was negative. His nerve conduction studies done in the second week after admission were reported normal. Magnetic resonance imaging (MRI) brain showed abnormal signal intensity involving medulla oblongata and posterior side of the pons and basal ganglia as shown in Fig. (1) and (2). In view of progression of symptoms and MRI findings, suggested the diagnosis of a demyelinating processes, which was consistent with a possibility of Bickerstaff brainstem encephalitis. Anti GQ1b was positive both in serum and CSF. This boy received a 2-day course of IVIG with total dose $2 \mathrm{gm} / \mathrm{kg}$. Patient showed dramatic 
improvement after one week in the form of improvement in dysarthria and dysphagia with regaining of normal consciousness level. At discharge, after three weeks of admission he was just having little difficulty in walking. Clinical and radiological follow-up after seven months was normal.

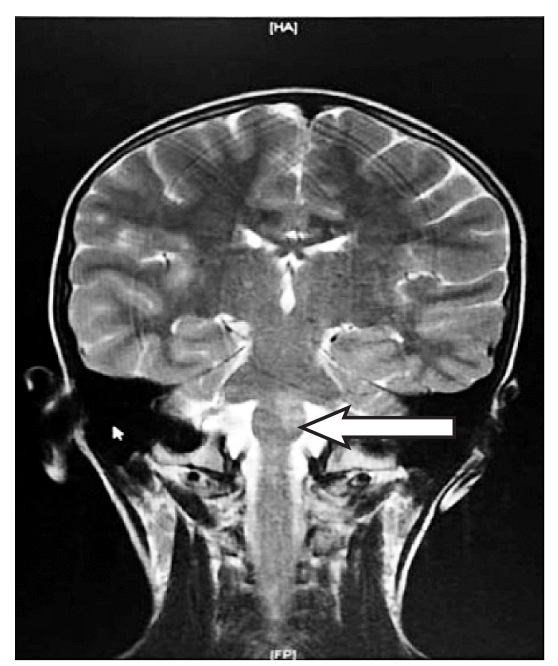

Fig. (1). T2-Weighted Brain Magnetic Resonance Imaging Showing (A) Hyperintensity in the Pons (arrow).

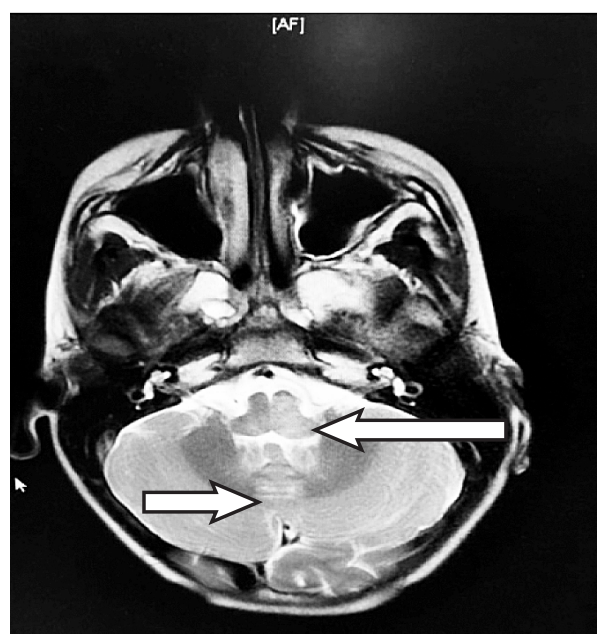

Fig. (2). T2-Weighted Brain Magnetic Resonance Imaging Showing (A) hyperintensity in the in the Pons (arrow) and Cerebellum (arrow).

\section{DISCUSSION}

In 1951 Bickerstaff \& Cloake described three cases of altered sensorium, ophthalmoplegia and ataxia, they also picked up lesion in the midbrain on MRI that was responsible for these clinical signs. International Pediatric Multiple Sclerosis Study Group described BBE as a type of Clinically Isolated Syndrome which is a post-infectious inflammatory demyelinating disorder. This CIS is the only one that is associated with encephalopathy.
BBE is defined as symptoms of acute ophthalmoplegia, ataxia, and disturbed consciousness, are the major manifestations. The clinical criteria for the diagnosis of BBE is; (1) Symmetric ophthalmoplegia and ataxia, (2) Disturbed conscious level or exaggerated deep tendon reflexes, and (3) strength in the limb muscles of grade four or five [5]. The clinical features of BBE is similar to those of the Miller Fisher syndrome (MFS); the common clinical signs are ophthalmoplegia, ataxia and CSF albumin-cytological dissociation [6]. However, the clinical criteria for the diagnosis of BBE must have disturbed conscious level or signs of upper motor neuron lesion, which show involvement of cortex and serious brainstem; the FS does not include these characteristics [7]. The exact mechanism of BBE is not well understood. However, there is general consensus that BBE is an autoimmune disorder triggered by infections. Eighty to ninety percent of the cases of MFS and GBS respectively and $68 \%$ of the cases with BBE present with ophthalmoplegia. Anti GQb1 antibodies are present in the early course of disese [8].

In our case, antibodies against mycoplasma was detected but titers were mildly raised moreover, there were no symptoms suggesting an infection at the time of admission. The presence of Anti-GQ1b IgG antibodies in the sera of the patients support the clinical diagnosis of BBE, Guillain Barre syndrome, and Miller Fisher Syndrome. Although anti-GQ1b antibodies have been reported in few post infectious disorders but most commonly reported in patients with $\mathrm{BBE}[9,10]$.

Brain imaging (MRI) detects the high intensity in the brain stem, thalamus, and cerebellum in $30 \%$ of the patients with BBE [11].

Brain MRI of our patient showed typical abnormal signals in the brain stem with positive anti gangliosides antibodies (anti GQ1b IgG) suggests the diagnosis of BBE. EEG study showed slow background without any abnormal epileptiform discharges indicating cerebrum involvement, consistent with altered consciousness. Nerve conduction studies were performed in the second week after admission which was reported normal. Because of frequent spontaneous recovery and rare type of disorder, currently there is lack of generalized consensus on the role of specific treatment for BBE.

Currently, IVIG and plasmapheresis are main treatment options for GBS and MFS and the prognosis is mostly good in patients with BBE [12].

\section{CONCLUSION}

$\mathrm{BBE}$ is a post infectious inflammatory demyelinating disorder that pediatric Neurologists rarely come across during their practice. Anti-GQ1b antibody seropositivity suggests possibility of BBE but most likely part of a spectrum of 
diseases under the umbrella known as Anti-Gq1b antibody syndrome. The resemblances between BBE, MFS, and GBS may often cause skepticism in reaching a diagnosis.

\section{CONFLICT OF INTEREST}

Declared none.

\section{ACKNOWLEDGEMENTS}

Declared none.

\section{REFERENCES}

[1] Bickerstaff ER, Cloake PC. Mesencephalitis and rhombencephalitis. Br Med J 1951; 2: 77-81. DOI: 10.1136/bmj.2.4723.77

[2] Bickerstaff ER. Brain-stem encephalitis: Further observations on a grave syndrome with benign prognosis. Br Med J 1957; (1): 1384-7. DOI: $10.1136 / \mathrm{bmj} .1 .5032 .1384$

[3] Koga M. Nationwide survey of patients in Japan with Bickerstaff brainstem encephalitis: Epidemiological and clinical characteristics. J Neurol Neurosurg Psychiatry 2012; 83(6): 1210-5. DOI: 10.1136/jnnp-2012-303060

[4] Kuwabara S. Fisher syndrome and Bickerstaff brainstem encephalitis. Brain Nerve 2015; 67: 1371-6.

[5] Shahrizaila N, Yuki N. Bickerstaff brainstem encephalitis and fisher syndrome: Anti-GQ1b antibody syndrome. J Neurol Neurosurg Psychiatry 2013; 84(5): 576-83. DOI: 10.1136/jnnp-2012-302824
[6] Ito M, Kuwabara S, Odaka M, et al. Bickerstaff's brainstem encephalitis and Fisher syndrome forma continuous spectrum: Clinical analysis of 581 cases. J Neurol 2008; 255 (5): 674-82. DOI: $10.1007 / \mathrm{s} 00415-008-0775-0$

[7] Saul RF. Neuro-ophthalmology and the anti-GQ1b antibody syndromes. Curr Neurol Neurosci Rep 2009; 9(5) : 379-83. DOI: $10.1007 / \mathrm{s} 11910-009-0055-0$

[8] Wakerley BR, Uncini A, Yuki N, GBS Classification Group. Guillain-Barr'e and miller fisher syndromes-new diagnostic classification. Nat Rev Neurol 2014; 10(9): 537-44. DOI: 10.1038/nrneurol.2014.138

[9] Susuki K, Yuki N, Hirata K. Fine specificity of anti-GQ1b IgG and clinical features. J Neurol Sci 2001; 185: (1): 5-9. DOI: 10.1016/S0022-510X(01)00464-6

[10] Suresh N, Schutt C, Tumbur A, et al. A case highlighting the phenotypic variant associated with Anti-GQ1B IgG spectrum disorder. Neurology 2017; 88(16): 194-5.

[11] Odaka M, Yuki N, Yamada M, et al. Bickerstaff's brainstem encepha litis: Clinical features of 62 cases and a subgroup associated with Guillain-Barré syndrome. Brain 2003; 126(10): 2279-90. DOI: 10.1093/brain/awg233

[12] Santoro JD, Lazzareschi DV, Campen CJ, et al. Pediatric Bickerstaff brainstem encephalitis: A systematic review of literature and case series. J Neurol 2018; 265(1): 141-50. DOI: $10.1007 / \mathrm{s} 00415-017-8684-8$ 